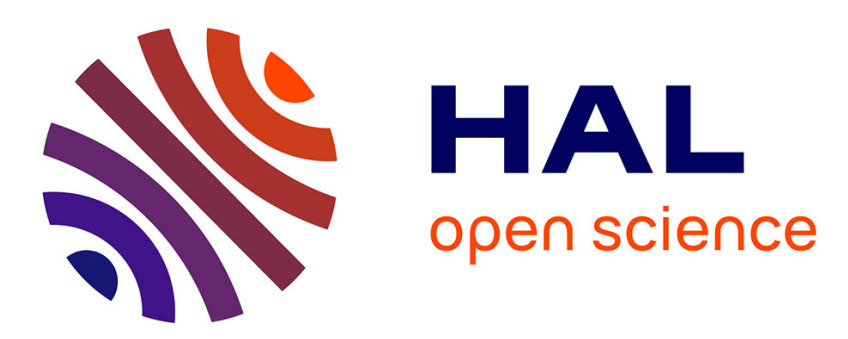

\title{
A MECHANISM FOR THE METAL INSULATOR TRANSITION AND VARIOUS PROPERTIES IN SAMARIUM COMPOUNDS
}

\author{
T. Kasuya
}

\section{- To cite this version:}

T. Kasuya. A MECHANISM FOR THE METAL INSULATOR TRANSITION AND VARIOUS PROPERTIES IN SAMARIUM COMPOUNDS. Journal de Physique Colloques, 1976, 37 (C4), pp.C4261-C4-265. 10.1051/jphyscol:1976445 . jpa-00216559

HAL Id: jpa-00216559

https://hal.science/jpa-00216559

Submitted on 1 Jan 1976

HAL is a multi-disciplinary open access archive for the deposit and dissemination of scientific research documents, whether they are published or not. The documents may come from teaching and research institutions in France or abroad, or from public or private research centers.
L'archive ouverte pluridisciplinaire HAL, est destinée au dépôt et à la diffusion de documents scientifiques de niveau recherche, publiés ou non, émanant des établissements d'enseignement et de recherche français ou étrangers, des laboratoires publics ou privés. 


\title{
A MECHANISM FOR THE METAL INSULATOR TRANSITION AND VARIOUS PROPERTIES IN SAMARIUM COMPOUNDS
}

\author{
T. KASUYA \\ Department of Physics, Tohoku University Sendai, Japan
}

\begin{abstract}
Résumé. - On a étudiẻ les états $4 \mathrm{f}$ dans les phases isolantes et métalliques de $\mathrm{EuB}_{6}$ et de $\mathrm{SmB} 6$. Dans notre modèle nous avons estimé de façon satisfaisante les propriétés de transport et les largeurs de raie RPE. Les transitions isolant-métal dans SmS dopé avec $Y$ ou avec As ont été étudiées en utilisant les concepts d'exciton magnétique et d'états d'impuretés magnétiques. L'accord avec l'expérience est excellent.
\end{abstract}

Abstract. - The $4 \mathrm{f}$ states for the insulator side $\operatorname{EuB}_{6}$ and the metallic side $\mathrm{SmB}_{6}$ are investigated. Transport properties and ESR line widths of Gd and $\mathrm{Eu}$ in $\mathrm{SmB}_{6}$ are evaluated satisfactorily on this model. Metal insulator transitions in $Y$ and As doped $\mathrm{SmS}$ are studied on the bases of the molecular magnetic exciton and molecular magnetic impurity state in excellent agreement with experiments.

1. Introduction. - In various $\mathrm{Sm}$ compounds, $\mathrm{Sm}$ ions change the valency from divalence to trivalence, which causes the complicated metal-insulator transition accompanied with various anomalous properties. In this sense, these compounds have been studied very intensively in the recent several years [1]. Among them, Sm-chalcogenides, $\mathrm{SmX}$, in which $\mathrm{X}$ means $\mathrm{S}$, Se or $\mathrm{Te}$, and $\mathrm{Sm}$-hexaboride, $\mathrm{SmB}_{6}$, have attracted much attention. In particular, $\mathrm{SmB}_{6}$ is the most convenient material to study the mechanism for the above transition because the rigid lattice is formed mainly by boron and thus only a small change of the volume is observed with the above transition. At first, in the present paper, we treat the mechanism for the metalinsulator transition in $\mathrm{SmB}_{6}$ based on the recent experimental results of our group and then treat that for SmS mostly doped with $Y$ and As worked by IBM group.

2. Physical picture for $\mathbf{S m B}_{6}$. - In rare earth borides, $\mathbf{R B}_{6}, \mathbf{R}$ and the molecule $B_{6}$, which is similar to the chalcogen $\mathrm{X}$ in $\mathrm{RX}$ and thus exists as $\mathrm{B}_{6}^{-}{ }^{-}$, forms the $\mathrm{CsCl}$ type lattice. Therefore, $\mathrm{EuB}_{6}$, in which $\mathrm{Eu}^{++}$is the stable state, should be insulator similar to EuX while other $R B_{6}$ should be metallic with one conduction electron per a rare earth atom. Recent remarkable improvement for the sample preparation now makes possible to produce very high purity samples so as to determine the Fermi surface of $\mathrm{LaB}_{6}$ very clearly [2]. Thus we now know that the bottoms of the conduction band are at X-points of the $\mathrm{e}_{\mathrm{g}}$ character with the band width of a couple of $\mathrm{e}$. $V$. The situation of $\mathrm{EuB}_{6}$ is, however, not simple because the existing samples are all metallic. This is due to the following facts. Recent experiment by Mercurio et al. [3] shows that the $4 \mathrm{f}$ levels are situated very closely to the bot- tom of the conduction band, may be several tenths of e. V., much closer than these in EuX. While in $\mathbf{R B}_{6}$, because of the rigid boron net work, some amount of the rare earth vacancies is always produced. In usual situation, this makes the sample p-type, such as Eu vacancies in $\mathrm{EuX}$. However, in $\mathrm{EuB}_{6}$, due to the small energy gap, this makes the sample n-type as shown in figure 1 . The vacancy has effectively two negative
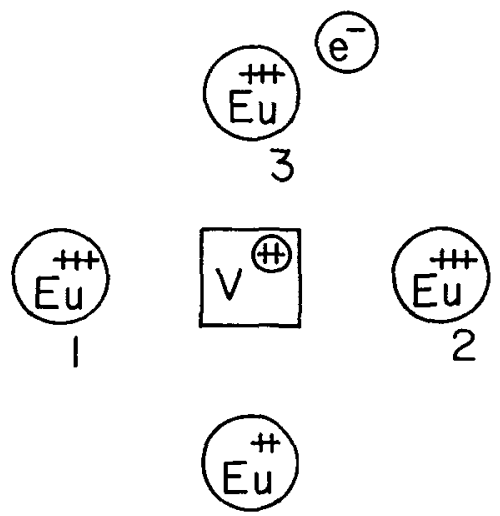

FIG. 1. - Schematic picture for Eu atoms around a vacancy. Boron sites are not shown.

charge and thus is compensated by producing two $\mathrm{Eu}^{+++}$ions as shown by sites 1 and 2 in figure 1 . Then, the potential at site 3 is raised by

$$
\Delta V=(2-\sqrt{2}) e^{2} / \varepsilon a,
$$

in which $\varepsilon$ is the effective dielectric constant with the value of the order one. By substituting, for example, $\varepsilon=4$ and $a=4.2 \AA$, we have $\Delta V=0.59 \mathrm{eV}$, which is nearly enough to push the $4 \mathrm{f}$ level at site 3 , or any other equivalent site, up to the bottom of the conduc- 
tion band. Thus, we expect that one Eu vacancy creates three $\mathrm{Eu}^{+++}$states and one conduction electron, which should be bound weakly at the above mentioned defect complex but delocalized very easily for a small concentration of the vacancies, may be the order of $10^{-3}$ to $10^{-4}$. In this way, EuB $_{6}$ can exist nearly always as the n-type degenerate semiconductor.

EuB $_{6}$ is ferromagnetic below about $15 \mathrm{~K}$. Our best samples show the residual resistivities $10^{-3} \Omega-\mathrm{cm}[4]$. The resistivities increase sharply at the Curie temperature $T_{\mathrm{c}}$ and become nearly constant, $5 \times 10^{-3} \Omega-\mathrm{cm}$, to the room temperature due to the strong scattering by the defect complexes and the $\mathrm{d}-\mathrm{f}$ exchange interaction. The Hall constant measurement at $4.2 \mathrm{~K}$ shows large contribution of the anomalous Hall constant. From the normal part, the carrier number is estimated to be $10^{-2}$ per Eu atom and the mobility $45 \mathrm{~cm}^{2} / \mathrm{V} \mathrm{s}$. In the paramagnetic region, it is difficult to separate the anomalous term from the normal term. However, the assumption of no anomalous term leads to a too small value of the carrier concentration. It is rather natural to assume that the carrier concentration is nearly temperature independent at least up to the room temperature and a relatively large amount of the anomalous Hall effect exists even in the paramagnetic region. Then the mobility in the paramagnetic region is estimated to be $10 \mathrm{~cm}^{2} / \mathrm{V} \mathrm{s}$. Note that the mobility of $\mathrm{LaB}_{6}$ is $80 \mathrm{~cm}^{2} / \mathrm{V} \mathrm{s}$ at the room temperature. This is important because in $\mathrm{SmB}_{6}$ Nickerson et al. [5] proposed a very unusual band scheme based on their Hall constant measurement assuming no anomalous Hall effect. This is discussed later.

In $\mathrm{SmB}_{6}, \mathrm{Sm}$-vacancies can be created as far as the one third of the $\mathrm{Sm}$ sites with very small change of lattice constant, that is $\mathrm{Sm}_{1-x} \square_{x} \mathrm{~B}_{6}$ for $0<x<1 / 3$ [6]. For $x=1 / 3$, all Sm should be trivalent. Detailed experimental study has been done in our group for various stoichiometries of $\mathrm{Sm}_{1-x} \square_{x} \mathrm{~B}_{6}$ [7]. All anomalous properties are explained consistently by the following model.

It is already well established that in nearly stoichiometric $\mathrm{SmB}_{6}$ the ratio of $\mathrm{Sm}^{++}$and $\mathrm{Sm}^{+++}$is about $4: 6$ to $3: 7$ and is temperature independent [8]. Therefore, the $4 \mathrm{f}$ levels are now pushed up to the middle of the conduction band and the Fermi level is just in the $4 \mathrm{f}$ levels. Note that in $\mathrm{SmX}$ the $4 \mathrm{f}$ levels are pushed up about $1.5 \mathrm{eV}$ from these of EuX. It is obviously impossible to treat the $4 \mathrm{f}$ levels by the usual one electron picture. The following picture may by one of the approximations for such states. The ground state of $\mathrm{Sm}^{++}$is the singlet, $S=3, L=3$ and $J=0$. Therefore we choose it the bases state, or the effective vacuum, and introduce the Fermion creation and annihilation operators $\beta_{v}^{+}$and $\beta_{v}$, respectively, in which the suffix $v$ means a state $J \neq 0$ for $\mathrm{Sm}^{++}$ or any state for $\mathrm{Sm}^{+++}$but hereafter, for simplicity, $v$ is restricted to the six states for $S=5 / 2, L=5$ and $J=5 / 2$ of $\mathrm{Sm}^{+++}$. Note that in the cubic environ- ment the $J=5 / 2$ states are split into the doublet and the quartet with the energy gap of the order of $100 \kappa$, in which $\kappa$ is the Boltzmann constant. Then the main term of the Hamiltonian may be written as

$$
\begin{aligned}
\mathscr{H}_{0}=\sum_{i k} \varepsilon_{i k} \alpha_{i k}^{+} \alpha_{i k} & +\sum_{v, n} \varepsilon_{v} \beta_{v n}^{+} \beta_{v n}+ \\
& +\sum \varepsilon_{v n, v^{\prime} m} \beta_{v n}^{+} \beta_{v^{\prime} m}+V_{n}\left(v, v^{\prime}\right)
\end{aligned}
$$

in which $\alpha_{i k}^{+}$and $\alpha_{i k}$ are the usual creation and annihilation operators for the conduction electron and the last term is an infinitely large correlation energy to avoid the state $\beta_{v n}^{+} \beta_{v^{\prime} n}^{+}$. Therefore, $\mathcal{H}_{0}$ corresponds to the extended Hubbard Hamiltonian. Note that to create a $\mathrm{Sm}^{+++}$, one electron is freed which we assumed to be put at the bottom of the conduction band so as to fix the energy scale. Therefore, $\varepsilon_{y}$ is negative and $\varepsilon_{i k}$ is measured from the bottom of the conduction band. Note also that the third term, the effective transfer of the $4 \mathrm{f}$ electron, comes mostly from the mixing with the valence bands and is important in EuX, an order of $0.1 \mathrm{eV}$, for the $4 \mathrm{f}$ band width [9], but should be much smaller in $\mathrm{SmB}_{6}$. The main terms of the interaction term may be written in the following simple form

$$
\begin{aligned}
\mathscr{H}_{1}=\sum v_{n}\left(i k, j k^{\prime}, v v^{\prime}\right) \alpha_{i k}^{+} & \alpha_{j k^{\prime}} \beta_{n v}^{+} \beta_{n v^{\prime}}+ \\
& +\sum V_{n v i k} \beta_{n v}^{+} \alpha_{i k}^{+}+\text {c. c. }
\end{aligned}
$$

in which the first term includes both the Coulomb type and the $\mathrm{d}-\mathrm{f}$ exchange type interactions and the second term means the main term for the d-f mixing, which is the order of a few hundreds $\kappa$ both for $\mathrm{SmB}_{6}$ and $\mathrm{SmX}^{9}$.

Because the $4 \mathrm{f}$ band width is very small, in the zeroth approximation, we can pick up the first two terms of $\mathscr{H}_{0}$ and the Coulomb term of $\mathscr{H}_{1}$, which may be approximated by $v\left(n_{c}\right) n_{c} n_{3}$ in which $n_{c}$ and $n_{3}$ are the number of the conduction electrons and that of $\mathrm{Sm}^{+++}$, respectively. This is the Hamiltonian treated by Falicov and Kimball [10] and, as the conduction band width is large, gives a nearly temperature independent values for $n_{\mathrm{c}}$ and $n_{3}$. In the next step, we treat the third and fourth terms of $\mathscr{H}_{0}$ following the spirit of Hubbard approximation [11]. We further simplify the approximation as follows. For the $v$-th state of $\mathrm{Sm}^{+++}$, the available sites to move around are $\left(c_{2}+c_{v}\right) \mathrm{N}$, in which $c_{2}$ and $c_{v}$ are the fractions of $\mathrm{Sm}^{++}$and the $v$-th state of $\mathrm{Sm}^{+++}$, respectively, and $N$ is the total $\mathrm{Sm}$ sites. Then the $v$-th band width is reduced by the factor $\left(c_{2}+c_{\nu}\right)$ while the density of state is kept constant. For the paramagnetic state, we have such six fold degenerate bands. When the d-f mixing term is taken into account, the conduction electrons near to the Fermi level mix strongly with the above mentioned $4 \mathrm{f}$ bands. From the symmetry consideration, no $5 \mathrm{~d}$ conduction electrons at the Fermi level are free from the d-f mixing, and as the density of state of the $4 \mathrm{f}$ bands is two order of magnitude larger than 
that of the conduction band, no conduction electron like states can exist near to the Fermi level. The situation is shown in figure 2 , in which the energy scale for the $4 \mathrm{f}$ bands are shown by the reversed sign for convenience. If we assume $c_{2}=0.4$, then, $\mathrm{n}$ the paramagnetic region, $C_{v}=0.1, c_{2}+c_{v}=0.5$ and the Fermi level is expected to be situated at the sharply falling part of the $4 \mathrm{f}$ bands. Then we immediately arrive the following conclusion that, similar to Pd [12], the susceptibility due to the $\mathrm{Sm}^{+++}$bands should increases gradually with increasing temperature and makes a broad peak at about a few $10 \mathrm{~K}$, while the coefficient for the linear $\mathrm{T}$ dependent term of the specific heat also increases gradually and makes a broad peak similar to the susceptibility. These behaviours are really observed experimentally $[5,7,13]$. The absolute values for the susceptibility and the specific heat are also consistent with the present picture of the narrow $4 \mathrm{f}$ bands with the band width of a few hundreds $\kappa$.

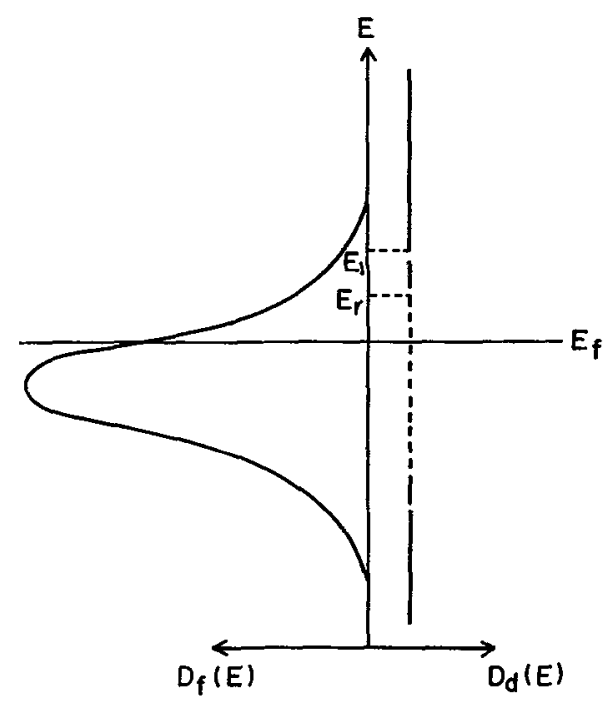

Frg. 2. - Density of states for the $4 \mathrm{f}$ character, $D_{P}(E)$, and the $5 \mathrm{~d}$ character, $D_{d}(E)$, near at the Fermi energy $E_{\mathfrak{f}}$ are shown schematícally. In the region given by a dotted line, the conduction band like states cannot exist due to the d-f mixing effect. The broken line part means the transient region to the conduction band in which $E_{1}$ corresponds to the boundary for the conduction phenomena while $E_{\mathrm{r}}$ corresponds to that for the relaxation mechanism. See the text.

At low temperature, the electrical conductivity is mostly due to the $4 \mathrm{f}$ bands. This should be similar to the impurity band conduction in the usual semiconductors and thus we use the formulation derived by Kasuya for that puporse [14]

$$
\sigma_{x}=\frac{\pi e^{2} \hbar}{\kappa T} \sum_{v} \int_{-\infty}^{\infty} \mathrm{d} E D_{v}^{2}(E) f_{1}(E) \zeta_{\nu}(E, E)
$$

in which $D_{v}(E)$ is the density of state for the $v$-th band, $f_{1}=f(1-f)$, where $f$ is the Fermi distribution function, and $\zeta_{v}(E, E)$ is the average of the square of the velocity matrix, $\left|\left\langle v_{1}\left|v_{x}\right| v_{2}\right\rangle\right|^{2}$, on the equi-energy surface $E$, where $v_{1}$ and $v_{2}$ are the eigenstates of the $v$-th band. The velocity operator $v_{x}$ was defined here as

$$
v_{x}=\frac{i}{\hbar} \sum_{n m v}\left(X_{m}-X_{n}\right) \varepsilon_{v n, v m} \beta_{v n}^{+} \beta_{v m}
$$

and, in the narrow band limit, $\zeta_{v}(E, E)$ may be evaluated to be

$$
\zeta_{v}(E, E)=\frac{a^{2} \Delta_{v}^{2}}{3 \hbar^{2} N_{v}}
$$

where $N_{v}=N\left(c_{2}+c_{v}\right), \Delta_{v}=\varepsilon Z\left(c_{2}+c_{v}\right)$, in which $\varepsilon$ is the nearest neighbour transfer energy and $Z$ is the number of the nearest neighbour sites, therefore six in the present case, and thus $\Delta_{v}$ means the effective band width for the $v$-th band. When the density of state is rewritten in the form $D_{v}(E)=\rho(E) N_{v} / \Delta_{v}$, where $\rho_{v}(E)$ should be about unity at the center of the band, the conductivity $\sigma_{v}$ is written finally in a simple form

$$
\sigma_{v}=\frac{\pi e^{2} a^{2}}{18 \hbar} N \rho_{v}\left(E_{\mathrm{f}}\right)
$$

or, by substituting $a=4.15 \times 10^{-8} \mathrm{~cm}$,

$$
\begin{gathered}
N=1.4 \times 10^{22} \mathrm{~cm}^{-3}, \\
\sigma_{v}=10^{3} \rho^{2}\left(E_{\mathrm{f}}\right) \quad(\mathrm{ohm}-\mathrm{cm})^{-1}
\end{gathered}
$$

and $\sigma$ is six times $\sigma_{v}$. Therefore, $\sigma$ can be near $10^{4}(\Omega-\mathrm{cm})^{-1}$ in maximum and this is really observed in $\mathrm{Y}$ doped SmS by Penney and Holtzberg [15]. However, in stoichiometric $\mathrm{SmB}_{6}, \sigma$ is about $5(\Omega-\mathrm{cm})^{-1}$ at $4.2 \mathrm{~K}[7,13]$ and is still decreasing gradually with decreasing temperature. This should be reckoned as follows. Similar to the usual impurity band, when the potential fluctuation exceeds the band width due to the translational energy, the $4 \mathrm{f}$ band states are localized. In $\mathrm{SmB}_{6}$, we expect that the translational $4 \mathrm{f}$ band width is only a few hundreds $\kappa$, much smaller than that in SmX. While, we always expect some $\mathrm{Sm}$ defects which cause the potential fluctuation. Therefore, the hopping type conduction is rather expected for the $4 \mathrm{f}$ band in $\mathrm{SmB}_{6}$. For the samples with more $S m$ vacancies or $R^{+++}$doping, the potential fluctuation is rather smeared out and the conductivity increases more than one order of magnitude [7]. In the high pressure phase of SmS [16], the potential fluctuation is expected to be relatively higher than that of $\mathrm{Y}$ doped sample and thus the conductivity decreases to the order of $10^{3}(\Omega-\mathrm{cm})^{-1}$. These are in the boundary between the metallic and the hopping $4 \mathrm{f}$ band conduction.

As temperature increases, the population on the conduction band increases and thus the conduction band conduction $\sigma_{\mathrm{c}}$ given by

$$
\sigma_{\mathrm{c}}=2 e \int D(E) \mu(E) f(E) \mathrm{d} E
$$


is observed, in which $\mu$ is the mobility. If we use the simplified model that $D(E)$ is a constant, $D_{\mathrm{c}}$, and $\mu(E)=0$ for $E<E_{1}$ and $\mu(E)=\mu_{\mathrm{c}}$ for $E>E_{1}$, the experimental curve above $4 \mathrm{~K}$ in $\mathrm{SmB}_{6}$ is well fitted by the following value; $E_{1}-E_{\mathrm{f}}=20 \%$, $\mu_{\mathrm{c}}=100 \mathrm{~cm}^{2} / \mathrm{V} \mathrm{s}$. and $D_{\mathrm{c}}=10^{34} \mathrm{erg}^{-1}=1.1$ state/ eV. Sm site.

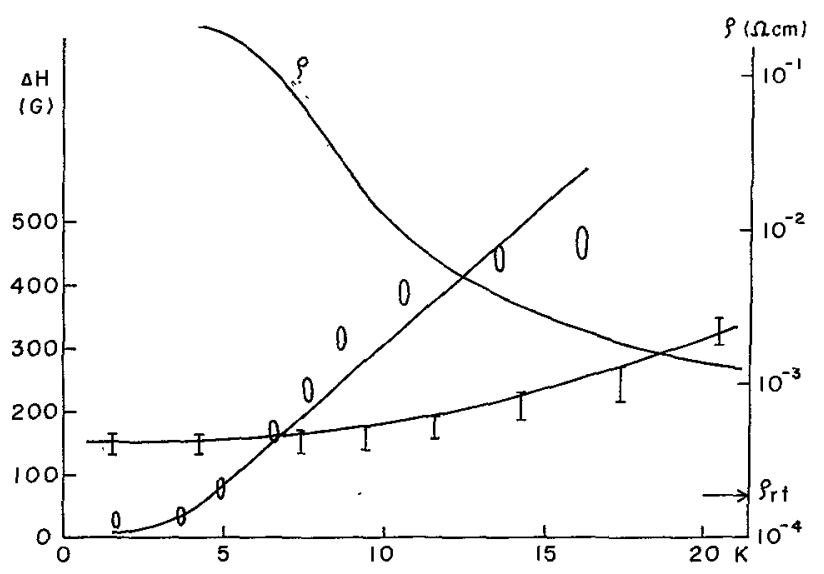

FIG. 3. - The Experimental values for the EPR line widths of $\mathrm{Gd}, \mathrm{O}$, and Eu, I, are shown as functions of temperature. Theoretical curves given in text are drawn by solid lines, respectively. Temperature dependence of the resistivity of $\mathrm{SmB}_{6}, \rho$, is also shown for comparison. The value at room temperature, $\rho_{\mathrm{rt}}$, is shown by the arrow.

EPR of dilutely doped $\mathrm{Gd}$ and Eu in various stoichiometric $\mathrm{SmB}_{6}$ has been done by Kojima [7]. The line width $\Delta H_{1}$ of $\mathrm{Gd}$ due to $T_{1}$ is shown in figure 3 . As the origin of the life time, the d-f exchange interaction is usually most important. When we apply it in the present system based on the present model, $\Delta H_{1}$ is given by

$$
\Delta H_{1}=\frac{\hbar}{g \mu_{\mathrm{B}} T_{1}}=\frac{4 \pi}{g \mu_{\mathrm{B}}} \int|Y(E) D(E)|^{2} f_{1}(E) \mathrm{d} E
$$

in which $\mu_{\mathrm{B}}$ is the Bohr magneton number and $I(E)$ the d-f exchange constant depending on the character of the conduction electron. Again by using a simplified model that $D(E)=D_{\mathrm{c}}, I(E)=0$ for $E<E_{\mathrm{r}}$ and $I(E)=I$ for $E>E_{\mathrm{r}}$, we obtain good agreement with experiment with the values ; $I=0.024 \mathrm{eV}$ and $E_{\mathrm{r}}=8 \%$. Note that $E_{\mathrm{r}}$ is smaller than $E_{1}$ because for the resonance relaxation mechanism the small mobility part has equal contribution and thus it pushes down the effective boundary of the conduction band.

The hyperfine splitting is large in $\mathrm{Eu}$ and thus the total line width $\Delta H$ is given by $\left(\Delta H_{0}^{2} \times \Delta H_{1}^{2}\right)^{1 / 2}$, in which $\Delta H_{0}$ is due to the hyperfine splitting and also the dipole field and is temperature independent while $\Delta H_{1}$ comes from $T_{1}$. Because Eu exists as the divalent ion, the amplitude of the conduction electron on $\mathrm{Eu}$ site is small and thus the effective d-f exchange constant I should be much smaller than that in Gd. There- fore, in $\mathrm{Eu}$, we rather expect the relaxation process through the $4 \mathrm{f}$ band to be more important. For this purpose, eq. (9) should be modified as follows.

$$
\begin{aligned}
& |D(E)|^{2} \rightarrow\left(J+\frac{1}{2}\right)\left|D_{v}(E)\right|^{2}=3\left|D_{v}\left(E_{\mathrm{f}}\right)\right|^{2} \\
& |I(E)|^{2} \rightarrow(g-1)^{2} J(J+1)\left|I_{3}(0)^{2}\right| / 3
\end{aligned}
$$

in which the $g$-factor for $J=2.5$ is $2 / 7$ and $I_{3}(0)$ is the sum of the exchange interaction between a Eu spin and the neighbour $\mathrm{Sm}^{+++}$spins. Because $I_{3}(0)$ is connected with the $g$-shift and the susceptibility, we can estimate $I_{3}(0)$ from these experimental values to be about $4 \kappa$. Then, to get best fit with experimental $\Delta \mathrm{H}$, the $4 \mathrm{f}$ band width $\Delta_{v}$ is determined to be $400 \rho_{v}\left(E_{\mathrm{f}}\right) \kappa$, which is very reasonable. In this way, the life times of $\mathrm{Gd}$ and $\mathrm{Eu}$ spins in $\mathrm{SmB}_{6}$ are explained satisfactorily both qualitatively and quantitatively on our model.

3. Metal-Insulator Transition in Doped SmS. - As the space is limited, we describe the mechanisms for the metal-insulator transitions in $\mathrm{Y}$ and $\mathrm{Sm}$ doped SmS very briefly.

In pure $\mathrm{SmS}$, the energy gap between the $4 \mathrm{f}$ levels and the bottoms of the conduction band at $X$ points is expected to be about $1.2 \mathrm{eV}$ for the Frank-Condon condition, no lattice distortion. Taking into account the lattice distortion effect, $0.5 \mathrm{eV}$ for optical phonon, $0.16 \mathrm{eV}$ for acoustic phonon and $0.07 \mathrm{eV}$ for the polaron effect on the conduction electron, the real gap is evaluated to be about $0.5 \mathrm{eV}$. Furthermore, by considering the single exciton formation, the gap, or the zero phonon line in the optical absorption, is reduced to be about $0.3 \mathrm{eV}$. This contradicts with Wachters conjecture [17], but is consistent with experiments by IBM group $\left({ }^{1}\right)$. However, as shown by Kasuya [18], the single exciton is not the most stable state but the molecular magnetic exciton, MME, probably three pairs of single exciton, is more stable. This means that once an exciton is created, the bound electron polarizes the near neighbour $4 \mathrm{f}$ spins through the d-f exchange interaction and thus digs a potential of $I S \sim 0.3 \mathrm{eV}$ for the second exciton electron, but, because of the three fold degeneracy of the conduction band, the kinetic energy loss of the fourth electron is more than the energy gain due to the $d-f$ exchange potential. Thus, the formation energy for such a MME is estimated to be about $0.1 \mathrm{eV} /$ single pair, When the pressure is applied, the $4 f-t_{2 g}$ energy gap shrinks in the ratio $-8 \mathrm{meV} / \mathrm{kbar}$ and thus the instability of the MME occurs at about $12 \mathrm{kbar}$. Actually, however, the first order transition occurs before it, at about $7 \mathrm{kbar}$.

When $\mathrm{Y}$, or any $\mathrm{R}^{+++}$, is doped, one conduction electron is trapped to each $\mathrm{Y}$ atom and forms the magnetic impurity state, MIS, which is essentially equal to the stable single ME. Therefore, around $\mathrm{Y}$,

(1) T. Penney and F. Holtzberg. Private communication. 
the total formation energy of MME is reduced by nearly $0.3 \mathrm{eV}$. However, single $\mathrm{Y}$ is still not enough to make the formation energy negative and the pair $Y$ atoms at least sitting on the next nearest neighbour sites is enough to make the MME, or in the present case the molecular MIS, to be instable, in which three neighbour $\mathrm{Sm}$ change to $\mathrm{Sm}^{+++}$thus five electrons are trapped in total. However, among them, two electrons are trapped so loosely that they are delocalized very easily and form free conduction electrons. The above picture explains the experimental facts [15] excellently.

When As is doped, the valence bands on the neighbouring $S$ are pushed up which causes the $4 \mathrm{f}$ levels on the neighbouring $\mathrm{Sm}$ to be pushed up through the p-f mixing effect, or the antibonding effect [9]. This is enough to push the $4 \mathrm{f}$ levels on the nearest neigbour $\mathrm{Sm}$-sites up to more than $0.1 \mathrm{eV}$ so that the MME instable. Now, around an As atom, six $\mathrm{Sm}^{+++}$and six electrons are formed among which three electrons are delocalized easily. This picture is again in excellent agreement with experiment [19]. The detail and more topics will be published in separate papers.

4. Conclusion. - The $4 \mathrm{f}$ states in $\mathrm{EuB}_{6}$ and $\mathrm{SmB}_{6}$ have been studied based on the extended Hubbard model. Transport properties and the EPR line widths of $\mathrm{Gd}$ and $\mathrm{Eu}$ in $\mathrm{SmB}_{6}$ are explained satisfactorily on the above model.

Metal-insulator transition mechanisms in $\mathrm{SmS}$, as well as $\mathrm{Y}$ and As doped $\mathrm{SmS}$, are studied based on the instabilities of the molecular magnetic exciton and the molecular magnetic impurity state in excellent agreement with experiments.

Valuable discussions and offering of unpublished data by M. Kasaya, K. Kojima and Y. Ishikawa are greatly appreciated. Valuable discussions, comments and kind hospitality during the stay in the IBM research center in 1975 by F. Holtzberg, S. Von Molnar and T. Penney are also greatly appreciated.

\section{References}

[1] For example, see the recent review article, VARMA, C. M., Rev. Mod. Phys. 48 (1976) 219, and the references in it.

[2] Private communication by IshIzAwa, $Y$.

[3] Mercurio, J. P., Etourneau, J., Naslain, R., Hagenmuller, P. and Goodenough, J. B., J. Solid State Chem. 9 (1974) 37.

[4] IshiKawa, Y. and KaSAYA, M., unpublished data.

[5] Nickerson, J. C., White, R. M., LeE, K. N., Bachman, R., Geballe, T. H. and Huld, G. W., Phys. Rev. 3 (1970) 2030.

[6] Ninhara, K., Bull. Chem. Soc. Japan 44 (1971) 963.

[7] Korma, K., Dr. Thesis, Tohoku University, 1976. Also unpublished data by KoIIMA, $K$. and KASAYA, $M$.

[8] See, for example, references 1 and 5 .

[9] KasuYa, T., IBM J. Res. Dev. 14 (1970) 214.

[10] Falicov, L. M. and Kimball, J. C., Phys. Rev. Lett. 22 (1969) 997.
[11] Hubbard, J. A 276 (1963) 238 and A 277 (1964) 237.

[12] For example, TaKahashi, T. and Shrmizu, M., J. Phys. Soc. Japan 20 (1965) 26.

[13] Menth, A., Buehler, E. and Geballe, T. H., Phys. Rey. Lett. 22 (1969) 295.

[14] Kasuya, T., J. Phys. Soc. Japan 13 (1958) 1096.

[15] Penney, T. and Holtzberg, F., Phys. Rev. Lett. 34 (1975) 322.

[16] Bader, S. D., Phillips, N. E. and MCWhan, D. B., Phys. Rev. B 7 (1973) 4686.

[17] Kaldis, E. and WACHTER, P., Solid State Commun. 11 (1972) 907.

[18] KasuYa, T., Solid State Commun. 18 (1976) 51.

[19] Von Molnar, S., Penney, T. and Holtzberg, F., J. Physique Colloq. 37 (1976) C4-239. 